\title{
1 Predicting metastasis with a novel 2 biophysical cell-adhesion force technique
}

3 Jessie $\operatorname{Gan}^{1}$, Zhao Zhihai ${ }^{29 *}$, Yu Miao ${ }^{39 *}$

$4 \quad{ }^{1}$ San Diego Jewish Academy, San Diego, CA, United States of America

$5 \quad{ }^{2}$ Department of Physics, National University of Singapore, Singapore

$6 \quad{ }^{3}$ Mechanobiology Institute, National University of Singapore, Singapore

7 9 These authors contributed equally to this work 


\section{Abstract}

9 Metastasis is widely accepted to be responsible for approximately $90 \%$ of all cancer deaths.

10 Current research on metastasis prediction often centers on gene sequencing; however, these

11 analyses must account for the complexity of gene regulation and rely on comprehensive datasets.

12 To investigate the process from a simpler, non-genomic angle, some studies indicate differences

13 in cell adhesion force, an important physical process in metastasizing cells. However, cell

14 adhesion force methods tend to focus on cell population approaches and therefore have their

15 drawbacks in cost or efficiency, rendering them impractical outside a research setting. In this

16 work, we test a novel and inexpensive bead-pipette assay to investigate the adhesion forces of

17 non-metastatic NIH3T3 cells and mutated RasV12 cells, a metastatic model cell line.

18 Control cells and RasV12 cells were evaluated with wound healing, spreading area, and focal

19 adhesion (FA) analysis assays. Then cells were tested by the novel bead-pipette assay, which

20 uses a fibronectin-coated bead and a glass micropipette to measure cell adhesion force using

21 Hooke's law.

22 The RasV12 cells had faster migration, polarized cell shape, and smaller FA area than control

23 cells. The RasV12 cells also exerted higher adhesion forces than control cells and a potential

24 force threshold was determined for distinguishing metastatic cells through a Receiver Operating

25 Characteristic (ROC) curve. An ROC curve was computed for all other assays and the bead-

26 pipette assay was shown to perform higher as a classifier than other assays.

27 The RasV12 cells had increased metastatic potential compared to control. The novel bead-pipette

28 assay showed potential as a classifier for determining metastasizing cells from non-metastatic 
29 cells. With further work, it may serve as a clinical diagnostic tool for cancer patients or as a

30 testbed to be used in the development of anti-metastatic drugs.

\section{Introduction}

Metastasis, the migration of cancer cells to a secondary tumor location, is a significant

33 contributor to cancer patient deaths(1). The onset and progression of metastasis is difficult to

34 predict and as yet, no universal prognostic metastasis marker has been identified. Most research

35 is focused on genomic markers through sequencing or microarray assays, but results are not

36 comprehensive and are typically cancer-type specific(2-5). Diagnostics using sequencing data

37 paired with machine-learning models, although getting faster and cheaper, still must account for

38 the complexity of the gene regulation of metastasis due to factors such as alternative splicing,

39 post-translational modifications, and protein processing(6,7). In addition, these diagnostic

40 models must be trained on immense and comprehensive datasets(8-10), which are tedious to

41 curate. Other tests include blood marker testing, CT scans, and MRI, which cannot diagnose

42 metastasis until the tumor has already metastasized(11).

Current literature indicates that cell adhesion force plays a major role in metastasis and is

44 influenced by cell genotype(12-15). Metastasis is defined by invasion and motility of the cancer

45 cell from the home base to a secondary location. Cell motility involves the integration of

46 multiple mechanical and chemical cues, many of which are driven by the adhesion of the cell to

47 the local extracellular matrix in its immediate neighborhood(16,17). Given that metastasizing

48 cells are known to move actively through the extracellular matrix and are affected by

49 environmental forces(18-20), it is possible that their adhesion forces will differ from those of

50 stationary cells. In the interest of simplifying and accelerating the detection of metastatic 
51 cancers, using adhesion force as a metric to differentiate non-metastatic and metastatic cells

52 could be a low-cost and high-throughput alternative to diagnostics based on genetic sequencing.

Metastasis is involved with cell adherence to extracellular matrix (ECM) and other cells

54 through protein complexes called focal adhesions (FAs)(21-23). FAs, the primary focus of this

55 work, are the interface for cells to interact and sense their local microenvironment, and they are

56 central hubs for mechanotransduction, ECM sensing, and directing cell migration(23-26). Sites

57 of FAs are initiated by the binding of integrin receptor proteins to ECM components and the

58 subsequent recruitment and clustering of cytoplasmic proteins and cytoskeletal elements.

59 Integrin, a transmembrane protein in FAs, has been shown to have a significant role in metastatic

60 processes $(22,27,28)$, and it binds to collagen and fibronectin (ECM components) through

61 hydrogen bonds and metal coordination(29-31). In stationary cells, the initial, nascent FAs

62 mature into larger, established FAs, which provide passive anchorage(32). However, in motile

63 cells, the cytoplasmic components generate a pulse of traction force upon the ECM substrate,

64 such as collagen or fibronectin (FN), then disassemble to form new FAs to propel the cell

65 forward(33).

Motile cells are often observed to be polarized and have distinct leading and trailing

67 edges $(36,37)$. The leading edge, characterized by the direction of movement, is formed by

68 protrusions controlled through actin polymerization. The leading edge is also characterized by

69 the FA turnover rate, the rate with which FAs assemble and disassemble to form new

70 FAs $(23,38)$. In motile cells, the leading edge may have a high turnover rate of FAs to continue

71 adhering to ECM as the cell moves forward(38). 
The idealized workflow is where patient biopsy samples would be directly tested in the

73 hospital lab to test patients' cells for metastasis, and thereby providing a valuable insight in the

74 progression of the disease. The adhesion of the cells obtained from the biopsy adhesion would be

75 tested by an adhesion force technique and classified based on a force threshold. In addition,

76 drugs tested for metastatic prevention can be evaluated for their relative effectiveness with the

77 adhesion force on certain extracellular substrates.

In order to use cell adhesion as a metric, there must be a consistent, versatile, and

79 affordable technique for measuring it. Many methods have been developed for quantifying cell

80 adhesion, such as traction force microscopy(39), centrifugal force assays(40), atomic force

81 microscopy(41), and single-cell aspiration(42). However, although many have advantages

82 including specific force observation and standard reproducibility, they also have disadvantages

83 such as low maximum forces and inaccurate modeling due to cell or chamber

84 deformation $(42,43)$. They also can be expensive or require an extensive number of cells,

85 rendering them inviable in a clinical setting where they could be assisting the diagnosis of cancer

86 patients.

To address the limitations of current methods for measuring cell adhesion, the Yan Jie lab

88 has developed the bead-pipette assay, a single-cell manipulation method of measuring adhesion

89 force implemented in this work. Its advantages include inexpensive materials, efficient

90 measurements, and precise control, and it shows potential to be not only applicable in a research

91 setting but also in a clinical and translational environment.

92 Currently metastasis accounts for an overwhelming majority of cancer deaths

93 worldwide(1), and an integral component in metastasizing cells is their adhesion strength based 
94 on the establishment, maturation rate, and deconstruction of FAs. In this work, we study the

95 potential to use cell adhesion as a metric for differentiating between metastatic and non-

96 metastatic cancers with a novel, biophysical assay. This is first investigated by first studying the

97 molecular interactions between integrins and FN, and the role of FAs in cell adhesion. Next we

98 developed an experiment to test the viability of the bead-pipette assay as a technique to measure

99 differentiating cell adhesion force, and we also assess other phenotypic aspects of the cells, such

100 as migration distance and FA size, to investigate their relationship to cell motility.

We hypothesized that the bead-pipette can effectively differentiate between metastatic

102 and non-metastatic cells, and that this approach is suitable for identifying metastasizing cells in

103 patient samples through cell adhesion force. The implementation of this technique in a clinical

104 setting could present a simple solution to the diagnosis of metastasis, by applying a physics

105 solution to a biological problem in an interdisciplinary application.

\section{Methods}

In this work, we use p53-knockout, mouse fibroblast NIH3T3 cells from the American

108 Type Culture Collection as control, non-metastatic cells. we use RasV12-transformed NIH3T3

109 cells as metastatic cells(44). The RasV12 cancer model activates metastatic-related pathways that

110 promote processes such as cell proliferation and invasion(44-46), making it suitable for this

111 work. The mutation results in a perpetually active Ras-GTP complex that is unable to be

112 inactivated by the Ras-GTPase activating proteins (GAPs)(47), and thereby continues to

113 upregulate metastatic-related pathways. 


\section{Wound Healing Assay}

We performed the wound healing assay to gauge the initial metastatic potential of the

117 cells. we first seeded the cells on collagen-coated glass with dividers from Ibidi GmbH

118 (Germany) for 24 hours until 100\% confluency was reached. Then we removed the dividers and

119 imaged the cells for another 24 hours afterwards as they moved to cover the gap from the

120 divider. We analyzed data at the eight-hour mark in the corresponding videos at 10x

121 magnification under a light microscope, and calculated the distance migrated in micrometers

122 through the Fiji ImageJ visualization program(48).

\section{Spreading Area}

We calculated the spreading area of the cells through imaging the cells with a light

125 microscope at 20x magnification. We photographed the cells at a low confluence and quantified

126 their area by outlining the cell membranes in Fiji ImageJ visualization program(48). We

127 performed this at $\mathrm{n}=20$ for each condition, for a total of 40 measurements.

\section{Focal Adhesions}

130 grown over two weeks were seeded overnight on small petri-dishes with a FN-coated glass well.

131 We used a 4\% paraformaldehyde solution to fix the cells, then $0.2 \%$ Triton to perforate the cell

132 membrane. We added Bovine Serum Albumin, a blocker to prevent non-specific binding, to the

133 fixed and permeabilized cells. We then added a primary antibody for paxillin from Cell

134 Signaling Technology and incubated the cells at $4{ }^{\circ} \mathrm{C}$ overnight. The next day we added a 
135 secondary antibody conjugated with Green Fluorescence Protein for the primary antibody. We

136 stained the nuclei with 4',6-diamidino-2-phenylindole (DAPI). All reagents were purchased from

137 Thermo Fisher Scientific (Waltham, MA) unless otherwise noted.

138 We performed fluorescence microscopy was performed with a Nikon A1R confocal microscope.

139 We visualized the cells under a 405 and $488 \mathrm{~nm}$ wavelength laser for the nuclei and anti-paxillin

140 antibody, respectively. We identified focal adhesions were identified through setting a brightness

141 threshold of 3320 gray level in a 16 bit image (gray level range of 0-65535), and then quantified

142 them by the "Analyze Particles" feature in Fiji ImageJ(48). FA measurements were taken for

$143 \mathrm{n}=20$ for each condition, for a total of 40 cells.

\section{Force Quantification - Bead Pipette Assay}

The force quantification bead assay is a novel force-measurement method that utilizes a

146 ECM-coated bead and a glass micropipette to measure cell adhesion force. Cells were seeded

147 overnight in a chamber composed of a polydimethylsiloxane cutout between two glass slides

148 coated in collagen. Before measurement, FN-coated beads made in the lab were added to the

149 chamber. The FN-coated beads were amino-coated polybeads, incubated with glutaraldehyde,

150 and then with FN.

$1 \mathrm{~mm}$ glass micropipettes were pulled to a fine point of about $2 \mu \mathrm{m}$ in diameter, and were

152 attached to a small water reservoir, through which suction into the pipette could be controlled by

153 changing the relative height to the microscope stage. The pipette was maneuvered into the cell

154 chamber with a micromanipulator, and was positioned to attach a bead by suction force. The cell

155 chamber was visualized with an Olympus Live EZ microscope under a 20x air lens. 


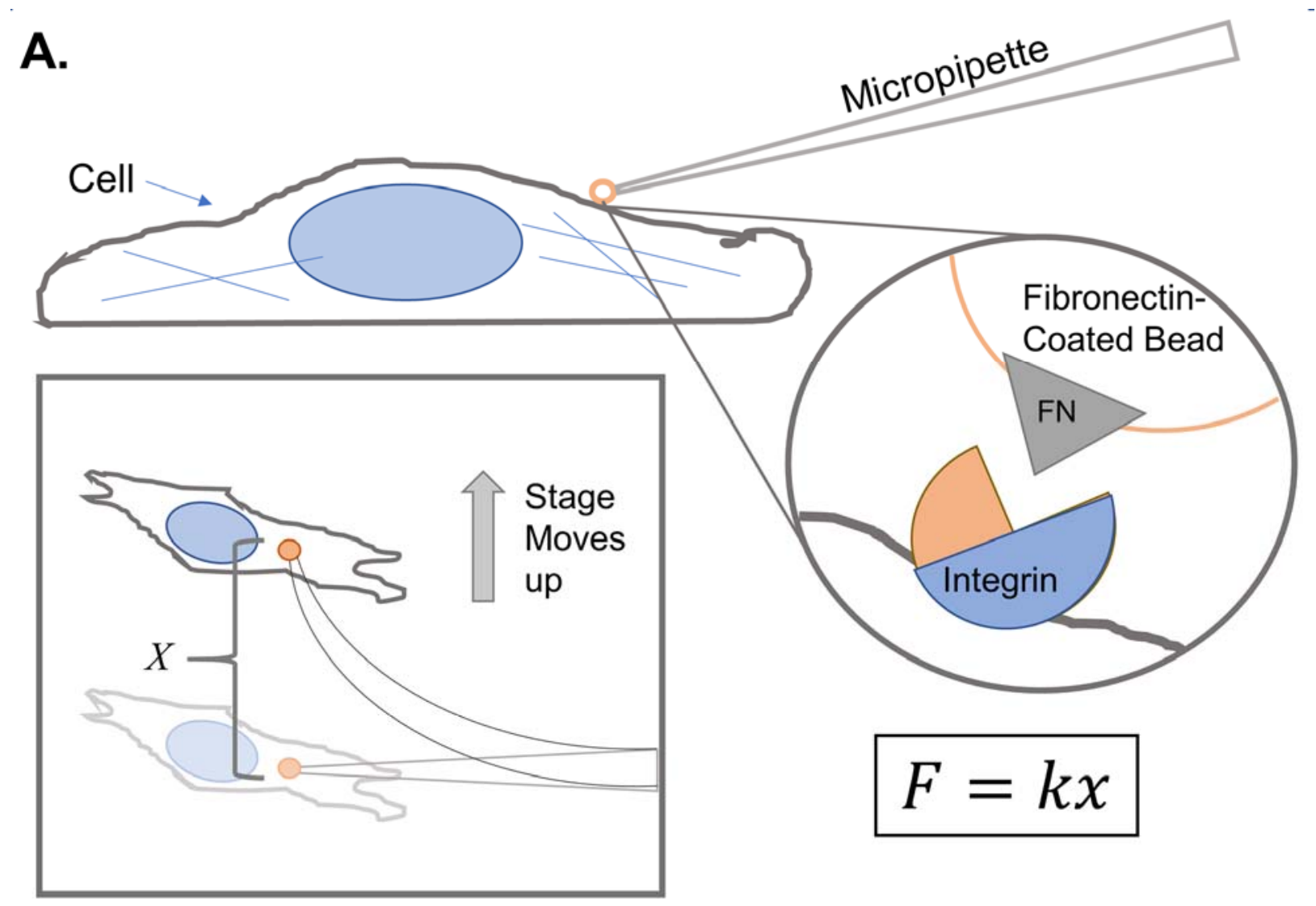

B.

Initial Contact

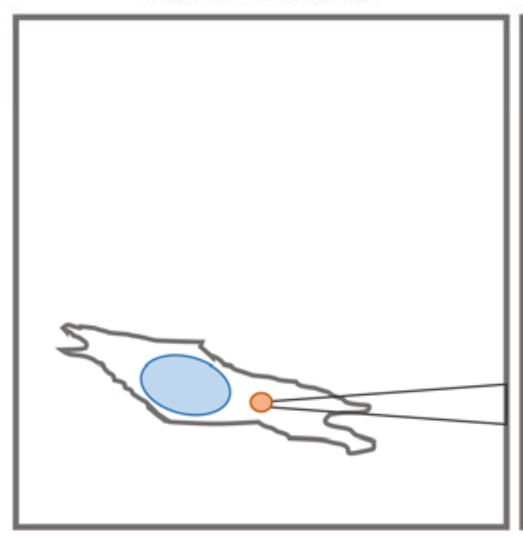

Stage Movement

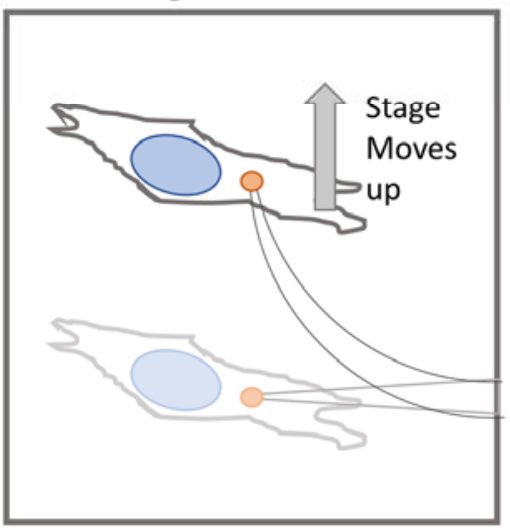

Pipette Deflection

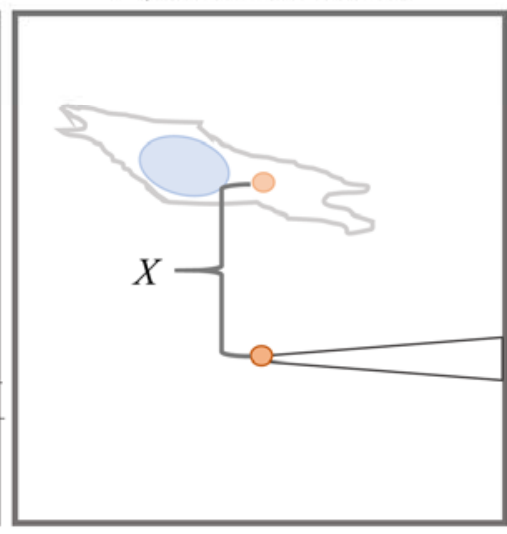

Fig 1. The bead-pipette method, using a flexible micropipette and FN-coated bead. A. The

force is quantified by contacting the bead with the cell, then measuring the deflection of the micropipette when the cell is moved away at $10 \mu \mathrm{m} / \mathrm{sec}$ in increments of $5 \mu \mathrm{m}$. Multiplying the deflection distance $(\mathrm{x})$ by the spring constant $(\mathrm{k})$ of the pipette gives the force needed to break the integrin-FN interaction. B. A sequential schematic of the bead-pipette assay steps from an aerial view. First the bead is lowered onto the surface of the cell at Initial Contact, then the stage 
163 is incrementally raised in the horizontal direction by the microscope. When the bead breaks

164 contact with the cell the distance is measured with image processing software Fiji ImageJ(43).

165 Using the micropipette to manipulate the FN-coated bead, cells were tested for adhesion

166 force by bringing the bead in contact with the cell surface and allowing the integrin-FN

167 interactions and FAs to form for 1 minute. Then the cell was moved in the horizontal direction at

168 a speed of $10 \mu \mathrm{m} / \mathrm{sec}$ by moving the microscope stage until the bead broke contact with the cell.

169 The process was photographed every $5 \mu \mathrm{m}$. After the bead broke contact with the cell surface,

170 the rebound distance of the pipette was measured in Fiji ImageJ(48) and the force was calculated

171 through the displacement and the spring constant of the micropipette, as shown in Figure 5A, B.

172 This procedure was performed at $n=20$ for each condition, for a total of 40 cells. The

173 micropipette spring constant was calculated in the lab. The corresponding spring constant and

174 distance were used to calculate the force values.

\section{Statistical Analysis}

For each assay the Student's T-Test(44) was calculated to determine if the difference

177 between the control and RasV12 cells was statistically significant. The difference was considered

178 statistically significant if the probability value (p-value) was below 0.05 .

179 To test the performance of adhesion force as a binary classifier of metastatic and benign

180 cells, the statistical analyses of an initial confusion matrix and a Receiver Operating

181 Characteristic (ROC) curve were computed in scikit-learn(45). The confusion matrix is a grid

182 that displays the percentage of true positives, true negatives, false positives, and false negatives

183 the classifier produced based on an arbitrary force threshold, which was initially chosen as the

184 lower standard deviation value of the RasV12 forces. To optimize an appropriate threshold and 
to compare the adhesion force as a classifier against other features measured, such as spreading

186 area, focal adhesions, and cell migration, ROC curves of the rate of false positives vs. the rate of

187 true positives were calculated. The area under the ROC curves and accuracy, precision, recall,

188 and Cohen's Kappa of the optimal threshold were calculated in Scikit-Learn(45).

\section{Results and Discussion}

The wound healing assay revealed that the RasV12 cells migrate farther than non-

192 metastatic cells (Fig 2A). However, the control cells tend to move together while the RasV12

193 cells move independently (Fig 2B). The control cells appear to have stronger cell-cell adhesion

194 through proteins such as cadherin, whereas the RasV12 cells are separated and seem to have

195 weaker cell-cell adhesion, which is another characteristic of metastatic cells.

196
B.
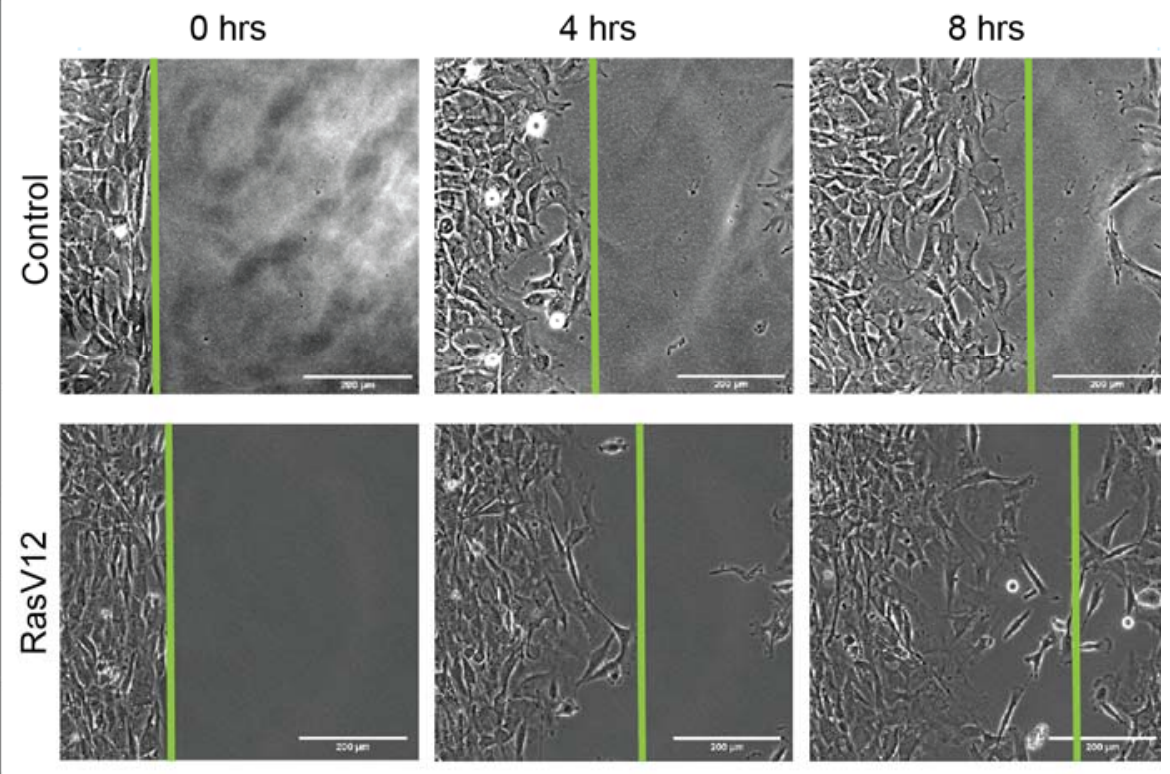

Fig 2A. Wound healing assay shows that the RasV12 cells had a higher migration potential than the non-mutated NIH3T3 cells. Over a period of 8 hours the RasV12 cells migrated farther. The control cells averaged at $230.45 \mu \mathrm{m}$ with \pm 26.76 error, and the RasV12 cells averaged at $311.35 \mu \mathrm{m}$ with \pm 34.62 error. Plot made with python data visualization package 
201 Matplotlib(46). B. Photographs show that the RasV12 phenotype moves farther than the control 202 phenotype.

203

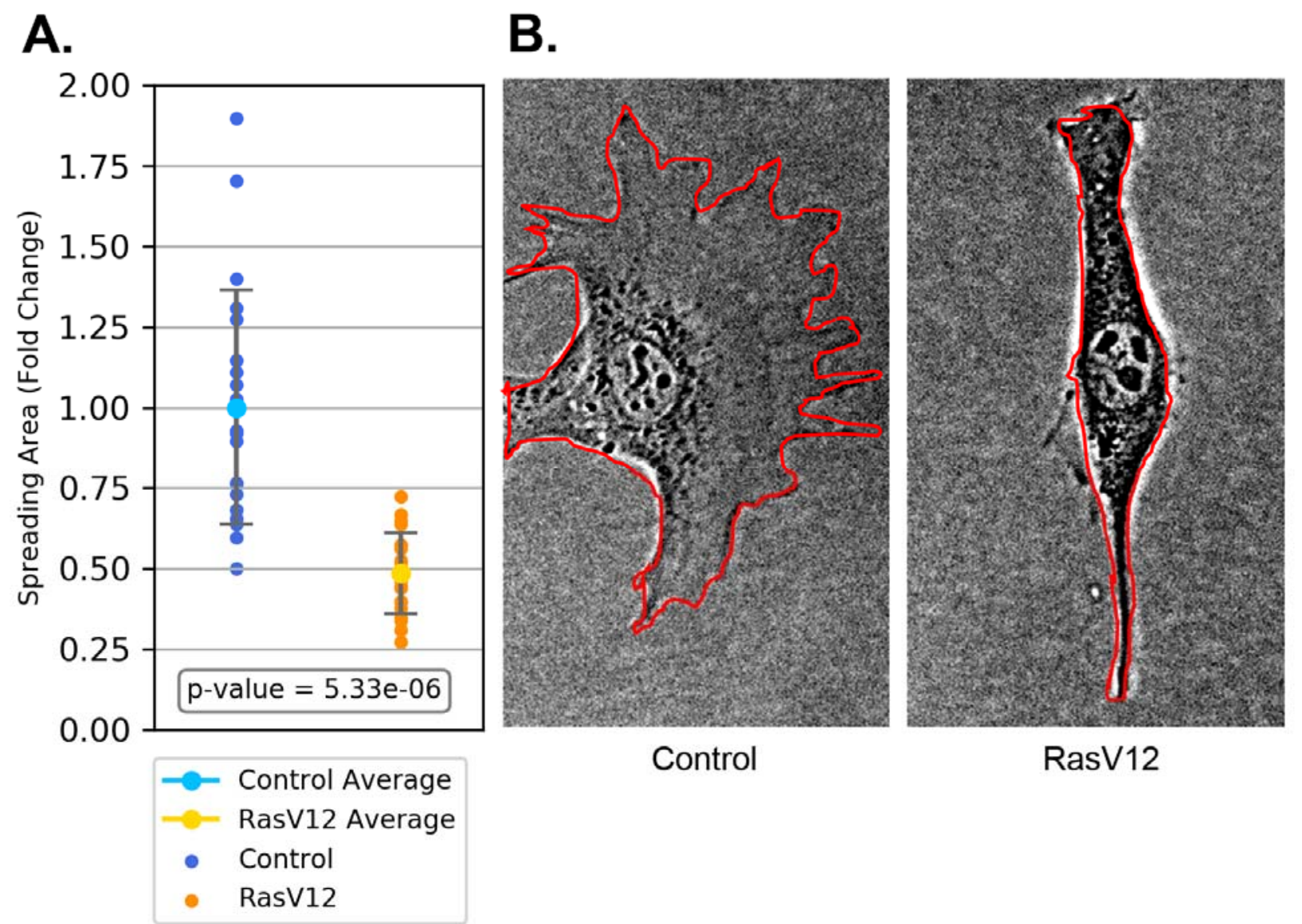

Fig 3A. The RasV12 cells show less spreading area than the control cells. The data is normalized to the mean of the control cell area. The plot was made with Matplotlib(46). B.

207 Spreading area outlines. These are representative examples of control and RasV12 cells with 208 their areas outlined in Fiji ImageJ(43).

The RasV12 phenotype cells have significantly less spreading area than the control cells

210 in Fig 3A. Representative cells are shown in Fig 3B, where the control cells are larger, with

211 tendrils of lamellipodium anchoring them over an extensive area. Their lack of polarization and

212 large area do not indicate a specific direction. In contrast, the RasV12 cells are thinner and

213 tapered, showing polarization of the FAs to a leading edge, a characteristic of migrating cells.

214 The leading edge would likely have nascent FAs forming, and the cell would direct a backwards 
215 force, pulling itself along the extracellular matrix, perpetually forming new FAs at its leading

216 edge and dissembling them at the trailing edge $(27,30)$. The polarization of the RasV12 cells

217 indicate their direction of movement, and result in a largely different cell shape than control

218 cells.

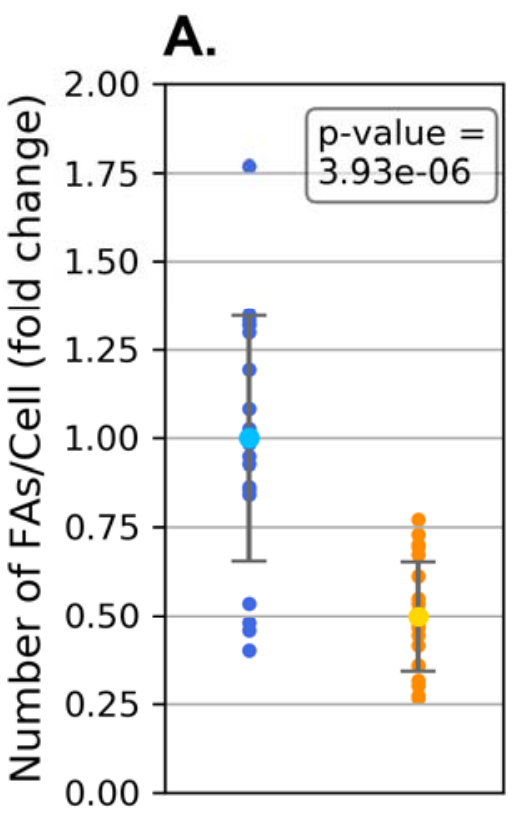

Control Average
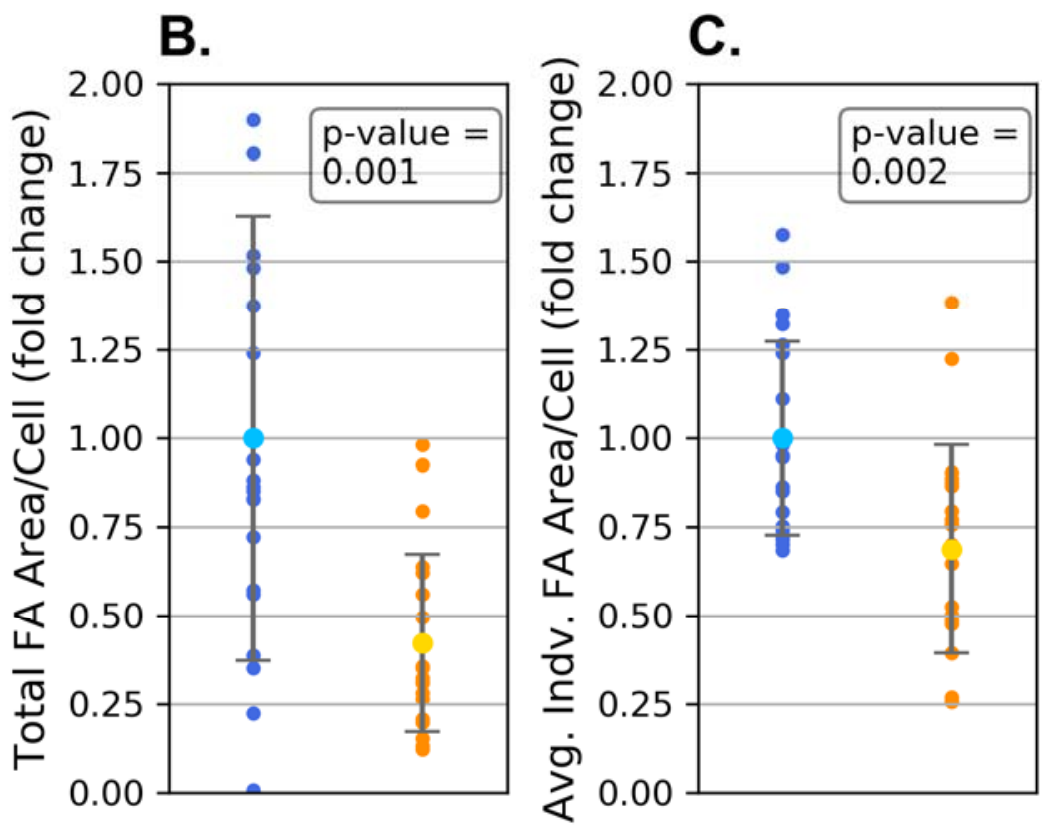

RasV12 Average

Control

RasV12

Fig 4. Focal adhesion quantitative analysis shows that RasV12 cells have less and smaller FAs than control. Control cells are in blue and RasV12 cells are in orange. All plots were made with Matplotlib(46) A. RasV12 cells have less total FAs than control cells. The data is normalized to the mean of the control number of FAs per cell. B. RasV12 cells have less total FA area than control cells. This data is normalized to the mean of the control cell focal adhesion size. C. RasV12 cells have smaller individual FA areas than control cells. This data is normalized to the average individual FA size for control cells.

The RasV12 cells overall showed fewer FAs (Fig 4A), coupled with less total FA area (Fig 4B) and smaller individual FA size (Fig 4C). This indicates less anchorage to their substrate than control cells. Motile cells will likely require fewer and smaller, nascent FAs; a moving cell needs to swiftly synthesize and deconstruct FAs. These results imply the migratory potential of the RasV12 cells. On the other hand, the control cells have larger FAs and more FA area. These 
232 features are characteristic of mature FAs, which are more established instead of transient(27).

233 More representative cells are shown in Fig 5.

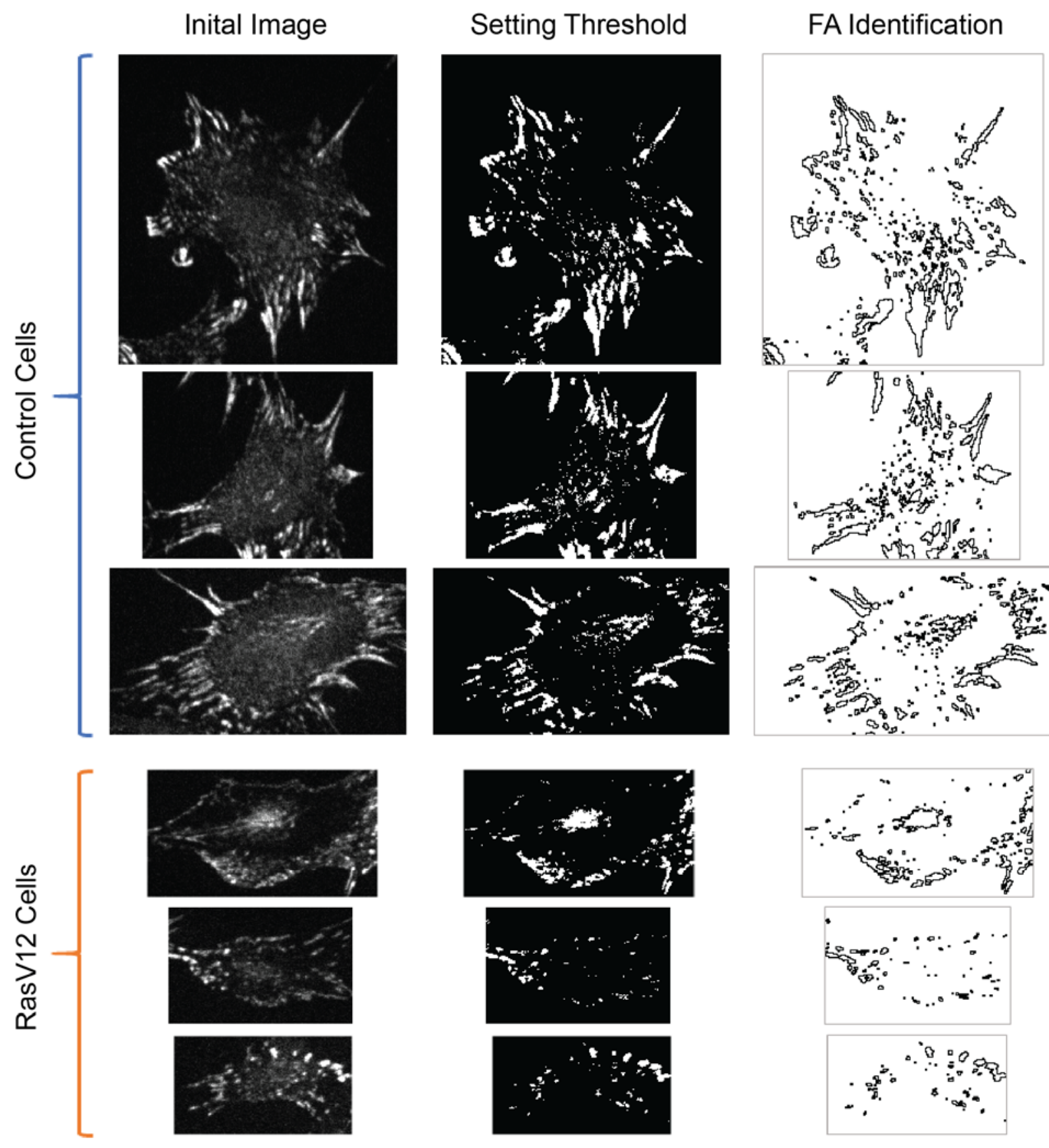

Fig 5. Representative cells are shown for each condition and each step in the process of calculating FAs through immunofluorescence assay in Fiji ImageJ(43). The original images were

237 automatically adjusted to view and section off individual cells. Then a standard threshold was set 238 and kept among all samples, and finally the "Analyze Particles" function was utilized to identify 239 individual FAs. 
A. B.

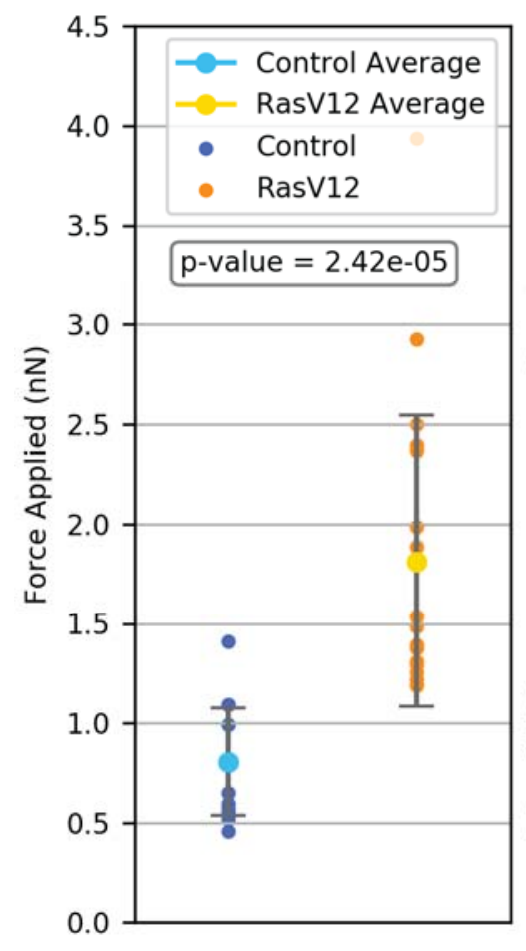

Contact
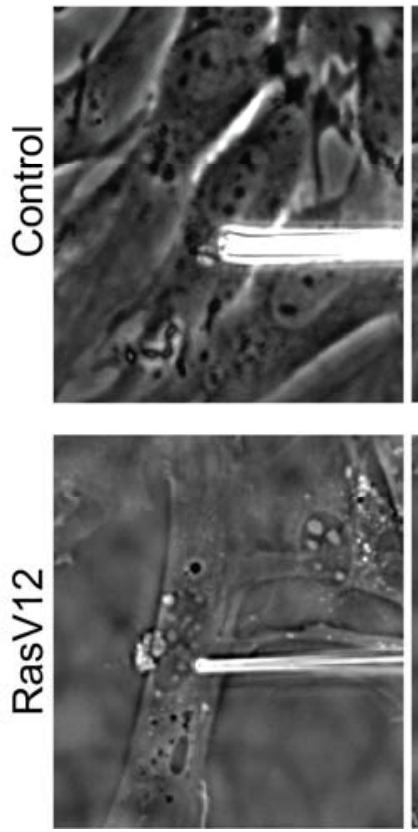

Break Point
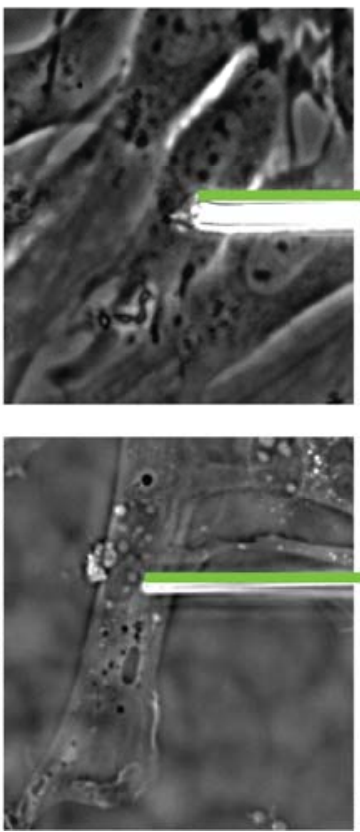

Deflection
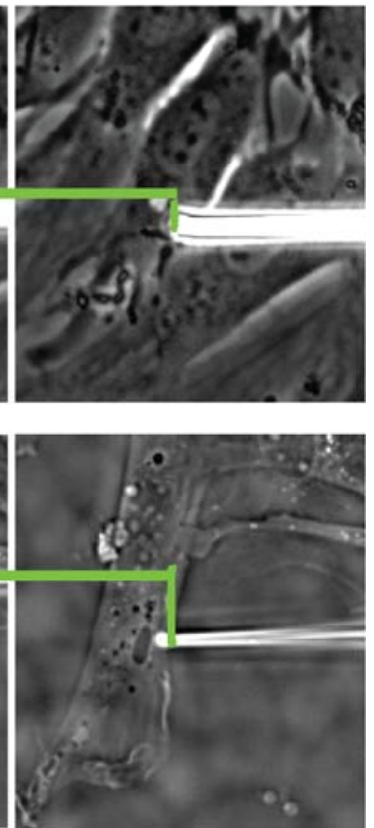

242 Fig 6A. RasV12 Cells' FAs exert higher force on FN ECM than control cells. The RasV12

243 phenotype cells have significantly higher force than the control cells. Plot was made in

244 Matplotlib(46). B. Difference in pipette deflection between control and RasV12 cells in the bead 245 assay. A larger distance contributes to a larger force exerted, in combination with the measured 246 stiffness, or spring constant.

In the bead-pipette assay, the RasV12 cells adhered to the FN-coated beads stronger than

248 the control cells (Fig 6A). On average, they adhered about twice as strong. From the FA analysis,

249 the RasV12 cells are shown to establish small FAs, and these nascent FAs appear to yield high

250 adhesion force regardless of their size. On the other hand, the control cells are likely synthesizing

251 FAs that form to maturity, however these are slowly assembled and deconstructed(23), and

252 therefore may exert low forces when initially forming. The stages of the bead-pipette assay are

253 shown in Fig 6B, where the bead is lowered onto the surface of the cell and a noticeably larger 
254 deflection is recorded for the RasV12 cells, indicating a higher force with similar micropipette

255 spring constants.
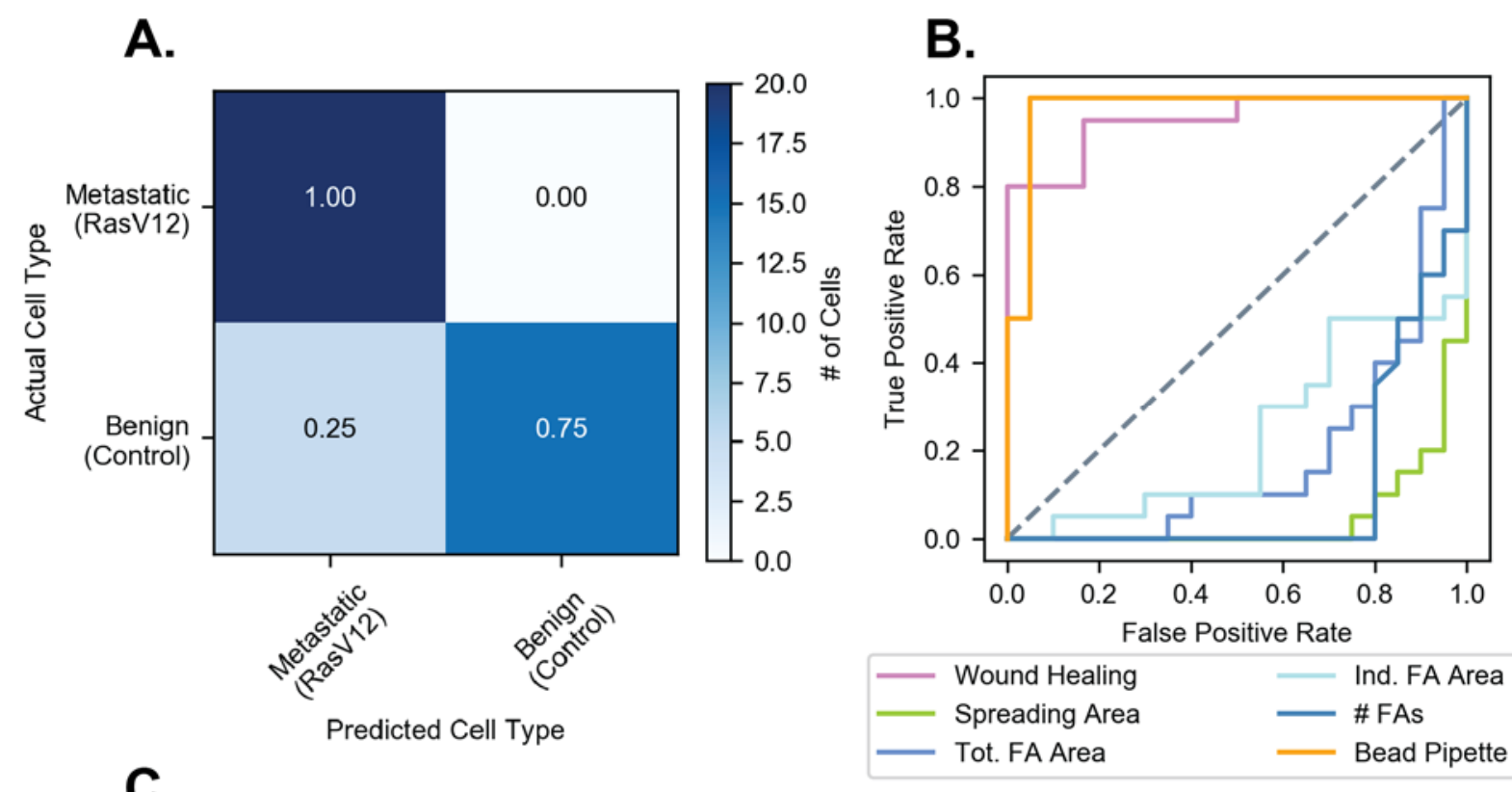

\begin{tabular}{|l|r|r|r|r|r|r|}
\hline Assay & $\begin{array}{l}\text { Area } \\
\text { Under } \\
\text { Curve }\end{array}$ & $\begin{array}{l}\text { Opt. } \\
\text { Threshold } \\
\text { (Opt. T) }\end{array}$ & $\begin{array}{l}\text { Accuracy at } \\
\text { Opt. T }\end{array}$ & $\begin{array}{l}\text { Precision } \\
\text { at Opt. T }\end{array}$ & $\begin{array}{l}\text { Recall at } \\
\text { Opt. T }\end{array}$ & $\begin{array}{l}\text { Cohen's } \\
\text { Kappa at } \\
\text { Opt. T }\end{array}$ \\
\hline Wound Healing & 0.95 & 290.24 & 0.84 & 1.00 & 0.75 & 0.69 \\
\hline Spreading Area & 0.05 & 2.90 & 0.50 & 0.50 & 1.00 & 0.00 \\
\hline FA Area & 0.19 & 0.12 & 0.48 & 0.00 & 0.00 & -0.05 \\
\hline Ind. FA Area & 0.24 & 2.58 & 0.50 & 0.50 & 1.00 & 0.00 \\
\# of Fas & 0.11 & 2.77 & 0.50 & 0.50 & 1.00 & 0.00 \\
\hline Bead Pipette & 0.98 & 1.19 & 0.95 & 0.95 & 0.95 & 0.90 \\
\hline
\end{tabular}

Fig 7. Statistical analysis of assays shows higher classifier performance of the bead-pipette assay. A. Example confusion matrix analysis for arbitrary bead pipette assay force threshold, plotted in Matplotlib(46). Cells were considered metastasizing if they exerted a force above the lower standard deviation value of the RasV12 cells (1.083 nN). Predictions were compared to cell genotypes.

B. Receiver Operating Characteristic (ROC) curves for each assay, calculated with Scikit-Learn(45) and plotted in Matplotlib(46). C. Matrix comparing area under ROC curves and accuracy, precision, recall, and Cohen's Kappa Coefficient(47) at the optimal threshold, determined through Youden's J Statistic(48). Values were calculated with ScikitLearn(45) and are colored relative to the range of values within each column.

Statistical analysis through a confusion matrix was performed for the adhesion force

267 classifier, where cells were considered metastasizing if their adhesion force was above the lower 
268 standard deviation value from average of the RasV12 forces (Fig 7A). This force threshold was

269 able to account for all RasV12 cells, but some control cells were able to exert a force in the range

270 of metastasizing cells, resulting in false positive values.

271 To find the optimal force threshold and compare the bead pipette assay as a classifier

272 against all other assays, ROC curves were plotted. The ROC curves in Fig 7B show that only the

273 bead-pipette assay and the wound healing assay can classify the metastatic and benign cells at a

274 standard above random classification. All other assays, while their differences are statistically

275 significant in terms of the Student's T-Test, perform lower than random classification. In Fig 7C,

276 the area under the ROC curves show that the bead-pipette assay performs slightly higher than the

277 wound healing, and both are markedly higher than other assays. Even at the optimal thresholds

278 for each assay, the accuracy, precision, and Cohen's Kappa - a statistic of classifier performance,

279 while considering random classification(47) - are notably higher for the bead-pipette and wound

280 healing assay. The only metric which the FA analyses and spreading area assays perform higher

281 is the recall, however the other metrics indicate that although these assays can classify all the

282 metastasizing cells correctly, they cannot effectively differentiate them from benign cells. The

283 full confusion matrices for all assays at optimal threshold are in S1 Fig.

285 that are often used to identify the hallmarks of metastatic cells. These statistical tests indicate that

286 while the adhesion force model still requires more testing and refinement over larger sample

287 sizes, using the bead-pipette assay to predict metastasis is promising. 


\section{Conclusion}

289 Assays in this work were able to identify several features of metastatic cells being expressed in

290 the RasV12 NIH3T3 cells used to model metastatic cancer cells. The bead-pipette assay was able

291 to quantify the difference between the control and RasV12 cells. The assay measured that the

292 control cells exerted less force than RasV12 cells on the same substrate over the same contact

293 time. The results also suggest that the turnover time may be significant in metastatic

294 mechanisms, as over the same period of time the RasV12 cells, with smaller FAs, were able to

295 adhere with more force, indicating a fast formation time.

296 The bead-pipette assay as a metastasis classifier has many advantages in this system. Concerning

297 its potential as a clinical diagnostic, the assay uses widespread and inexpensive laboratory

298 materials. To compare, AFM cantilevers and microscopes are expensive (on the order of tens of

299 thousands of US dollars) and require specialized training, as well as can have technical

300 drawbacks due to positional and time-based complications.

301 In addition, the bead-pipette assay measures individual cells, and only requires one seeding in

302 order to get significant results. In other methods such as hydrodynamic shear or centrifugal force

303 techniques, many cells are needed in order to produce significant results, as these techniques

304 typically measure the force needed to displace $50 \%$ of the cells. However, since the bead-pipette

305 assay measures each cell individually, fewer cells on the scale of a tumor biopsy would likely be

306 sufficient.

307 With respect to research, the bead-pipette assay also has advantages for studying cell adhesion

308 mechanisms. FA turnover time is an important factor in this work, however, most current

309 techniques of force quantification detach cells with pre-established FAs. In contrast, the bead- 
310 pipette assay controls the contact time and is primarily invested in measuring nascent FAs; the

311 assay gives insight into turnover rate.

312 In addition, the assay inflicts minimal deformation on the cell, allowing for repeated

313 measurements over a period of time. A contrasting example is the micropipette aspiration

314 technique, which displaces the cell from an adhered surface through suctioning the cell from a

315 surface. However this method can tear the cell membrane and can only be performed once per

316 cell. Other population-based methods also cannot repeat measurements on the same cells, as they

317 displace a certain number of cells and may damage them while displacing them.

318 Lastly, the bead-pipette assay allows the researcher to observe the cell and perform the adhesion

319 force measurement simultaneously. Although seemingly insignificant, this feature is not

320 commonly available in all methods such as centrifugation, and may be important for observing

321 particular phenotypes of interest. In this work, observing the cells from the measurement led to

322 preliminary analysis, and also allowed recognition of cells in an undesirable growth phase, for

323 example, apoptosis.

324 In conclusion, the novel bead-pipette assay has the potential to be a viable diagnostic tool for

325 distinguishing patient metastatic cells based on adhesion force. The RasV12 cells have displayed

326 multiple characteristics of metastasizing cells such as faster cell migration, polarized cell shape,

327 smaller FA area, and less FA numbers compared to control cells. Unlike other methods, the

328 bead-pipette assay is able to also account for turnover time of FA synthesis, and has shown that

329 the RasV12 cells have faster turnover time to account for cell motility. Due to the simplicity of

330 the technique and the novelty of the measurement, the bead-pipette assay has potential as an 
331 effective and accessible method of force quantification that applies a physical solution to a

332 biological problem.

\section{Acknowledgements}

334 This work was funded by the Mechanobiology Institute. In addition, the authors would like to

335 acknowledge Professor Yan Jie for integral feedback and review on this work.

\section{References}

337 1. Seyfried TN, Huysentruyt LC. On the origin of cancer metastasis. Crit Rev Oncog. 2013;18(1-2):43-73.

2. Achary PMR, Zhao H, Fan Z, Gogineni S, Pulijaal VR, Herbst L, et al. A candidate metastasis-associated DNA marker for ductal mammary carcinoma. Breast Cancer Res. 2003 Apr;5(2):R52.

3. Liu Z, Cheng X, Zhang L, Zhou J, Deng D, Ji J. A panel of DNA methylated markers predicts metastasis of pNOM0 gastric carcinoma: a prospective cohort study. Br J Cancer. 2019 Oct 1;121(7):529-36.

4. Sperlich A, Balmert A, Doll D, Bauer S, Franke F, Keller G, et al. Genetic and immunological biomarkers predict metastatic disease recurrence in stage III colon cancer. BMC Cancer. 2018 Dec;18(1):998.

5. Luo X, Qiu Y, Jiang Y, Chen F, Jiang L, Zhou Y, et al. Long non-coding RNA implicated in the invasion and metastasis of head and neck cancer: possible function and mechanisms. Mol Cancer. 2018 Dec;17(1):14.

6. Polewko-Klim A, Lesiński W, Mnich K, Piliszek R, Rudnicki WR. Integration of multiple types of genetic markers for neuroblastoma may contribute to improved prediction of the overall survival. Biol Direct. 2018 Jan;13(1):17.

7. Tan MS, Chang S-W, Cheah PL, Yap HJ. Integrative machine learning analysis of multiple gene expression profiles in cervical cancer. PeerJ. 2018 Jul 25;6:e5285.

8. Rauschert S, Raubenheimer K, Melton PE, Huang RC. Machine learning and clinical epigenetics: a review of challenges for diagnosis and classification. Clin Epigenetics. 2020 Dec;12(1):51.

9. Park SB, Chung CK, Gonzalez E, Yoo C. Causal Inference Network of Genes Related with Bone Metastasis of Breast Cancer and Osteoblasts Using Causal Bayesian Networks. J Bone Metab. 2018;25(4):251.

10. Dias R, Torkamani A. Artificial intelligence in clinical and genomic diagnostics. Genome Med. 2019 Dec;11(1):70.

11. Dinnes J, Ferrante di Ruffano L, Takwoingi Y, Cheung ST, Nathan P, Matin RN, et al. Ultrasound, CT, MRI, or PET-CT for staging and re-staging of adults with cutaneous melanoma. Cochrane Skin Group, editor. Cochrane Database Syst Rev [Internet]. 2019 Jul 1 [cited 2020 Nov 5]; Available from: http://doi.wiley.com/10.1002/14651858.CD012806.pub2 
12. Fuhrmann A, Banisadr A, Beri P, Tlsty TD, Engler AJ. Metastatic State of Cancer Cells May Be Indicated by Adhesion Strength. Biophys J. 2017 Feb;112(4):736-45.

13. Osmani N, Follain G, García León MJ, Lefebvre O, Busnelli I, Larnicol A, et al. Metastatic Tumor Cells Exploit Their Adhesion Repertoire to Counteract Shear Forces during Intravascular Arrest. Cell Rep. 2019 Sep;28(10):2491-2500.e5.

14. Mekhdjian AH, Kai F, Rubashkin MG, Prahl LS, Przybyla LM, McGregor AL, et al. Integrin-mediated traction force enhances paxillin molecular associations and adhesion dynamics that increase the invasiveness of tumor cells into a three-dimensional extracellular matrix. Yap A, editor. Mol Biol Cell. 2017 Jun;28(11):1467-88.

15. Spencer A, Spruell C, Nandi S, Wong M, Creixell M, Baker AB. A high-throughput mechanofluidic screening platform for investigating tumor cell adhesion during metastasis. Lab Chip. 2016;16(1):142-52.

16. Welch DR, Hurst DR. Defining the Hallmarks of Metastasis. Cancer Res. 2019 Jun 15;79(12):3011-27.

17. Scott KE, Rychel K, Ranamukhaarachchi S, Rangamani P, Fraley SI. Emerging themes and unifying concepts underlying cell behavior regulation by the pericellular space. Acta Biomater. 2019 Sep;96:81-98.

18. Mouw JK, Yui Y, Damiano L, Bainer RO, Lakins JN, Acerbi I, et al. Tissue mechanics modulate microRNA-dependent PTEN expression to regulate malignant progression. Nat Med. 2014 Apr;20(4):360-7.

19. Kumar S, Weaver VM. Mechanics, malignancy, and metastasis: The force journey of a tumor cell. Cancer Metastasis Rev. 2009 Jun;28(1-2):113-27.

20. Ricca BL, Venugopalan G, Furuta S, Tanner K, Orellana WA, Reber CD, et al. Transient external force induces phenotypic reversion of malignant epithelial structures via nitric oxide signaling. eLife. 2018 Mar 21;7:e26161.

21. Geiger B, Spatz JP, Bershadsky AD. Environmental sensing through focal adhesions. Nat Rev Mol Cell Biol. 2009 Jan;10(1):21-33.

22. Eke I, Cordes N. Focal adhesion signaling and therapy resistance in cancer. Semin Cancer Biol. 2015 Apr;31:65-75.

23. Lock JG, Wehrle-Haller B, Strömblad S. Cell-matrix adhesion complexes: Master control machinery of cell migration. Semin Cancer Biol. 2008 Feb;18(1):65-76.

24. Plotnikov SV, Pasapera AM, Sabass B, Waterman CM. Force Fluctuations within Focal Adhesions Mediate ECM-Rigidity Sensing to Guide Directed Cell Migration. Cell. 2012 Dec;151(7):1513-27.

25. Riveline D, Zamir E, Balaban NQ, Schwarz US, Ishizaki T, Narumiya S, et al. Focal Contacts as Mechanosensors: Externally Applied Local Mechanical Force Induces Growth of Focal Contacts by an mDia1-dependent and ROCK-independent Mechanism?. J Cell Biol. 2001;153:11.

26. Balaban NQ, Schwarz US, Riveline D, Goichberg P, Tzur G, Sabanay I, et al. Force and focal adhesion assembly: a close relationship studied using elastic micropatterned substrates. Nat Cell Biol. 2001 May;3(5):466-72.

27. Hamidi H, Ivaska J. Every step of the way: integrins in cancer progression and metastasis. Nat Rev Cancer. 2018 Sep;18(9):533-48.

28. Tennenbaum T, Yuspa SH, Grover A, Castronovo V, Sobel ME, Vainada Y, et al. Extracellular Matrix Receptors and Mouse Skin Carcinogenesis: Altered Expression Linked to Appearance of Early Markers of Tumor Progression. :12. 
29. Guo W, Giancotti FG. Integrin signalling during tumour progression. Nat Rev Mol Cell Biol. 2004 Oct;5(10):816-26.

30. Nagae M, Re S, Mihara E, Nogi T, Sugita Y, Takagi J. Crystal structure of $\alpha 5 \beta 1$ integrin ectodomain: Atomic details of the fibronectin receptor. J Cell Biol. 2012 Apr 2;197(1):13140 .

31. Luo B-H, Carman CV, Springer TA. Structural Basis of Integrin Regulation and Signaling. Annu Rev Immunol. 2007 Apr;25(1):619-47.

32. Beningo KA, Dembo M, Kaverina I, Small JV, Wang Y. Nascent Focal Adhesions Are Responsible for the Generation of Strong Propulsive Forces in Migrating Fibroblasts. J Cell Biol. 2001 May 14;153(4):881-8.

33. Pollard TD, Borisy GG. Cellular Motility Driven by Assembly and Disassembly of Actin Filaments. Cell. 2003 Feb;112(4):453-65.

34. Humphrey W, Dalke A, Schulten K. VMD: Visual molecular dynamics. J Mol Graph. 1996 Feb;14(1):33-8.

35. Harrington B. Draw Freely | Inkscape [Internet]. Inkscape. [cited 2020 Nov 9]. Available from: https://inkscape.org/

36. Etienne-Manneville S, Hall A. Integrin-Mediated Activation of Cdc42 Controls Cell Polarity in Migrating Astrocytes through PKC $\square$. :10.

37. Cau J. Cdc42 controls the polarity of the actin and microtubule cytoskeletons through two distinct signal transduction pathways. J Cell Sci. 2005 Jun 15;118(12):2579-87.

38. Vicente-Manzanares M. Cell migration at a glance. J Cell Sci. 2005 Nov 1;118(21):4917-9.

39. Colin-York H, Fritzsche M. The future of traction force microscopy. Curr Opin Biomed Eng. 2018 Mar;5:1-5.

40. Reyes CD, García AJ. A centrifugation cell adhesion assay for high-throughput screening of biomaterial surfaces: Centrifugation Cell Adhesion Assay. J Biomed Mater Res A. 2003 Oct 1;67A(1):328-33.

41. Dufrêne YF, Ando T, Garcia R, Alsteens D, Martinez-Martin D, Engel A, et al. Imaging modes of atomic force microscopy for application in molecular and cell biology. Nat Nanotechnol. 2017 Apr;12(4):295-307.

42. Christ KV, Turner KT. Methods to Measure the Strength of Cell Adhesion to Substrates. J Adhes Sci Technol. 2010 Jan;24(13-14):2027-58.

43. Khalili A, Ahmad M. A Review of Cell Adhesion Studies for Biomedical and Biological Applications. Int J Mol Sci. 2015 Aug 5;16(8):18149-84.

44. Campbell PM, Der CJ. Oncogenic Ras and its role in tumor cell invasion and metastasis. Semin Cancer Biol. 2004 Apr;14(2):105-14.

45. Fernandez-Medarde A, Santos E. Ras in Cancer and Developmental Diseases. Genes Cancer. 2011 Mar 1;2(3):344-58.

46. Zenonos K. RAS signaling pathways, mutations and their role in colorectal cancer. World $\mathbf{J}$ Gastrointest Oncol. 2013;5(5):97.

47. Bos JL, Rehmann H, Wittinghofer A, Colell A, Ricci J-E, Tait S, et al. GEFs and GAPs: Critical Elements in the Control of Small G Proteins. :1.

48. Schindelin J, Arganda-Carreras I, Frise E, Kaynig V, Longair M, Pietzsch T, et al. Fiji: an open-source platform for biological-image analysis. Nat Methods. 2012 Jul;9(7):676-82.

49. Student. The Probable Error of a Mean. Biometrika. 1908 Mar;6(1):1.

50. Pedregosa F, Varoquaux G, Gramfort A, Michel V, Thirion B, Grisel O, et al. Scikit-learn: Machine Learning in Python. Mach Learn PYTHON. :6. 
461 51. Hunter JD. Matplotlib: A 2D Graphics Environment. Comput Sci Eng. 2007;9(3):90-5.

462 52. Maître J-L, Heisenberg C-P. Three Functions of Cadherins in Cell Adhesion. Curr Biol. 4632013 Jul;23(14):R626-33.

464 53. Padmanaban V, Krol I, Suhail Y, Szczerba BM, Aceto N, Bader JS, et al. E-cadherin is required for metastasis in multiple models of breast cancer. Nature. 2019 Sep 19;573(7774):439-44.

54. Cohen J. A Coefficient of Agreement for Nominal Scales. Educ Psychol Meas. 1960 Apr;20(1):37-46.

469 55. Youden WJ. Index for rating diagnostic tests. Cancer. 1950 Jan;3(1):32-5.

\section{Supporting Information}

472 S1 Fig. Confusion matrices for all assays at optimal thresholds. Optimal thresholds and

473 corresponding confusion matrices were calculated in Scikit-Learn(45) and plotted in

474 Matplotlib(46).

475

476 S1 Table. Wound healing assay data of cell migration over 8 hrs.

477 S2 Table. Spreading area assay data of cell area.

478 S3 Table. Focal Adhesion analyses data for Focal Adhesion area and size.

479 S4 Table. Bead-Pipette assay data for cell adhesion force. 